\title{
Patterns of antropyloric motility in fed healthy preterm infants
}

\author{
B B Hassan, R Butler, G P Davidson, M Benninga, R Haslam, C Barnett, J Dent, \\ T I Omari
}

See end of article for authors' affiliations

Correspondence to: Dr Omari, Centre for Paediatric and Adolescent Gastroenterology,

Women's and Children's Hospital, North Adelaide, SA 5006, Australia;

taher.omari@adelaide.edu.au

Accepted 30 April 2002

\begin{abstract}
Background: Antropyloric motility is important for regulation of gastric emptying and has not been adequately characterised in premature infants.

Aim: To evaluate fed patterns of antropyloric motility in premature infants.

Subjects: Forty three healthy premature infants, 30-38 weeks of postmenstrual age.

Methods: Postprandial antropyloric motility was measured using a micromanometric feeding assembly (outer diameter $1.8 \mathrm{~mm}$ ) incorporating a pyloric sleeve sensor. The occurrence of isolated pyloric pressure waves (IPPWs) and antral pressure wave sequences (PWSs) was characterised. Sequences were further classified as being antegrade, synchronous, antegrade-synchronous, and retrograde according to the direction of propagation.

Results: A total of 7289 pressure wave events were recorded, 48\% IPPWs and $52 \%$ PWSs (18\% antegrade, $12 \%$ synchronous, $13 \%$ antegrade-synchronous, $2 \%$ retrograde, and $7 \%$ undefined). IPPWs predominated in the first postprandial hour, peaking at 30-60 minutes. PWSs predominated in the period after one hour postprandially. Mean (SEM) half gastric emptying time was 42 (4) minutes.

Conclusions: Monitoring of antropyloric motor patterns in healthy premature infants indicates that the neuroregulatory mechanisms responsible for the coordination of antropyloric motility and gastric emptying are well developed by 30 weeks of postmenstrual age.
\end{abstract}

f $\mathrm{n}$ premature infants, the early establishment of enteral feeding is beneficial for growth and development and is associated with reduced postnatal morbidity. ${ }^{1}$ Problems such as feed intolerance and/or gastro-oesophageal reflux are common in these babies and may be caused by abnormalities of gastric function, possibly in association with immaturity of gastric neuroregulatory control mechanisms.

Studies of gastric function in premature infants have used measurements of the rate of gastric emptying, gastric pacemaker activity by surface electrogastrography, and low resolution perfusion manometry. In premature infants as young as 30 weeks gestation, gastric half emptying times have been shown to vary from 17 to 72 minutes depending on the feed volume, type, and energy content. ${ }^{23}$ One recent electrogastrography study reported a significantly higher proportion of abnormal gastric pacemaker activity in infants less than 29 weeks gestation. ${ }^{4}$ Previous evaluations of antroduodenal motility in premature infants used manometric techniques, which limited the recording of antral motor patterns to one or two very widely spaced $(2.5-4 \mathrm{~cm}$ apart $)$ side holes. ${ }^{5-7}$ Furthermore, the exact position of these side holes relative to the pylorus was not precisely determined, and pyloric motor events could not be recorded because of the absence of a sleeve sensor.

High resolution manometric techniques have been widely used in the assessment of antropyloric motor function in adults using an array of 8-10 closely spaced side holes straddling the pylorus and a sleeve sensor to measure localised pyloric pressures. These studies in adults have shown that gastric motility is highly complex and that in the fed state the spatial and temporal coordination of these events changes substantially over time. During the early phase of gastric emptying the antrum is largely quiescent, and proximal gastric tone, in conjunction with isolated pyloric pressure waves (IPPWs), regulates flow of gastric contents through the gastric outlet. Over time and as the stomach empties, IPPWs are gradually replaced by coordinated patterns of propagated antral pressure wave sequences (PWSs), which facilitate mixing and pump gastric contents into the duodenum. ${ }^{8}$

Micromanometric techniques have previously enabled assessment of oesophageal body and lower oesophageal sphincter motility in healthy premature infants. ${ }^{9}{ }^{10}$ The aim of this study was to use micromanometric techniques to record patterns of antropyloric motility in healthy premature infants older than 30 weeks of postmenstrual age (PMA) and then to compare these recordings with those previously reported in adults.

\section{METHODS}

\section{Subjects}

Pyloric motility studies were attempted in 43 preterm infants ( 14 boys, 29 girls) from the special care baby unit at the Women's and Children's Hospital who were healthy for relative gestational age and were receiving full enteral bolus feeds. The protocol for this study had been approved by the research ethics committee of the hospital, and written informed consent was obtained from the parents. Mean (SEM) PMA was 35 (0.34) weeks (30-33 weeks, $\mathrm{n}=9$; 34-36 weeks, $\mathrm{n}=24$; 37-38 weeks, $\mathrm{n}=10$ ), and mean (SEM) weight was 2130 (75) g (range 1170-3100). No infants had known gastrointestinal problems or feeding difficulties or were receiving drugs that would interfere with normal gastrointestinal function. During the study, the infants were fed $5-85 \mathrm{ml}(3-31 \mathrm{ml} / \mathrm{kg})$ of either non-fortified expressed breast milk $(\mathrm{n}=37)$ or infant formula $(\mathrm{n}=6)$. The mean (SEM) time taken to administer the feed was 10 (4), 15 (3), and 17 (6) minutes for 30-33, 34-36, and 37-38 week infants respectively.

Abbreviations: IPPW, isolated pyloric pressure wave; PWS, pressure wave sequence; PMA, premenstrual age; TMPD, transmucosal potential difference 


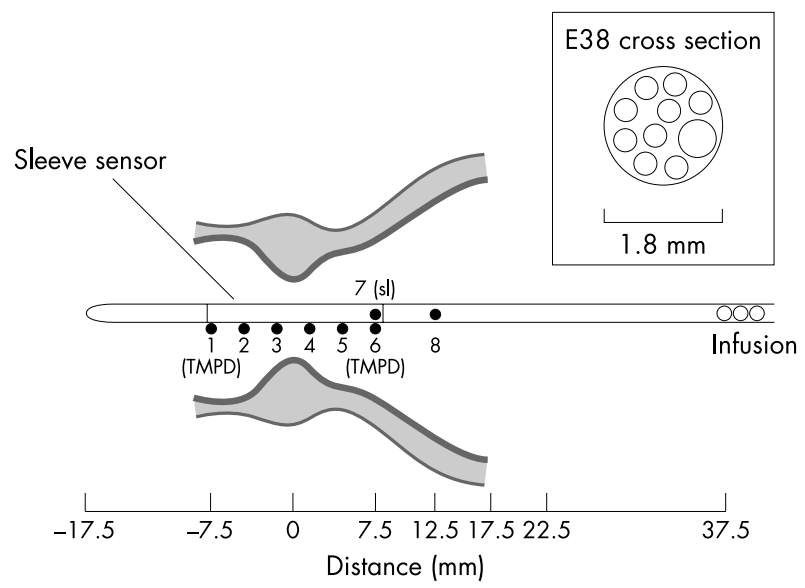

Figure 1 Schematic diagram showing side hole and sleeve (sl) positioning of the micromanometric feeding catheter used to record antropyloric motility. The transmucosal potential difference (TMPD) monitoring method allowed very accurate positioning of the sleeve relative to the pylorus. During studies, antral pressures were always recorded with at least four side holes - that is, side holes $4-6$ and 8 .

\section{Manometric technique}

Patterns of antropyloric motility were recorded with a micromanometric assembly that incorporated a $1.5 \mathrm{~cm}$ long sleeve sensor in parallel with seven perfused side hole sensors spaced at $3-5 \mathrm{~mm}$ intervals (fig 1 ). Unlike studies conducted in adults, tip weights were not used to assist transpyloric passage of the assembly.

Accurate positioning of the assembly across the pylorus was achieved by use of feedback from dual point transmucosal potential difference (TMPD) recording. ${ }^{11}$ To enable TMPD measurement across the pylorus, side holes 1 and 6 (located at the sleeve margins, fig 1 ) were perfused with sterile degassed saline $(0.9 \% \mathrm{NaCl}, 300 \mathrm{mOsm})$, creating a salt bridge between the gut lumen and the $\mathrm{Ag} / \mathrm{AgCl}$ electrodes located within the fluid pathway between the pressure transducers and the assembly luer connectors. TMPD signals were referenced to body potential by an $\mathrm{Ag} / \mathrm{AgCl}$ skin surface electrode. In practice, the gastric electrical potential was usually within the range 0 to $-50 \mathrm{mV}$, and the duodenal electrical potential was usually within the range 0 to $+50 \mathrm{mV}$. Accurate positioning of the sleeve across the pylorus was indicated by a TMPD gradient of $10 \mathrm{mV}$ or more between the stomach and duodenum. During studies, the assembly was positioned such that antral pressures were always recorded with at least four side holes-that is, side holes 4-6 and 8.

The sleeve and side holes $2-5$ and 8 were perfused with sterile degassed water. Throughout the study the manometric assembly perfusion delivered volumes not exceeding $12 \%$ of the infants daily fluid intake. To control the total amount of fluid infused, the perfusion rate was varied from 0.01 to 0.04 $\mathrm{ml} / \mathrm{min}$ by altering the perfusion pump driving pressure from 25 to $100 \mathrm{kPa}$. Even at these low perfusion rates, six of the infants, all 30-32 weeks PMA (1170-1620 g) were considered to be too small for performance of a full length study and were therefore only studied for 30 minutes postprandially; the remaining infants were all studied for two hours postprandially.

Analogue pressure transducer and TMPD signals were amplified and filtered with a BME 1600 polygraph (Department of Biomedical Engineering, Women's and Children's Hospital, North Adelaide, South Australia). Data acquisition and analysis were performed on a Macintosh Quadra 700, with software based on National Instruments' Labview (MAD software, Royal Adelaide Hospital; C Malbert) and Acqknowledge v3.0 (Biopac Systems Inc, Goleta, California, USA).

\section{Measurement of the rate of gastric emptying}

Gastric emptying was measured using the previously validated $\left[{ }^{13} \mathrm{C}\right]$ octanoic acid breath test. ${ }^{12-14}\left[{ }^{13} \mathrm{C}\right]$ Octanoic acid (Cambridge Isotope Laboratories, Cambridge, Massachusetts, USA; $50 \mu \mathrm{l}$ ) was added to the feed, which was immediately agitated to ensure maximal dispersion. After feeding, the rate of appearance of ${ }^{13} \mathrm{CO}_{2}$ in the breath is proportional to the rate of gastric emptying and is calculated using an established non-linear regression model. ${ }^{15}$ Breath was sampled through a 5Fr silastic tube inserted into the right naris. Breath samples were collected before feeding (baseline readings) and at five minute intervals for 30 minutes and then at 15 minute intervals for 210 minutes for a total breath collecting time of 240 minutes. We analysed breath samples using an isotope ratio mass spectrometer for ${ }^{13} \mathrm{CO}_{2}$ content. The percentage dose of ${ }^{13} \mathrm{CO}_{2}$ expired over time was fitted to standard mathematical formulae using a non-linear regression model. These formulae allowed calculation of the half gastric emptying time $\left(\mathrm{GEt}_{1 / 2}\right)$.

\section{Study protocol}

At the beginning of the study the infants were intubated with the manometric assembly, which was advanced until the TMPD recording side holes indicated that the sleeve was straddling the pyloric sphincter. The distal sleeve margin was positioned within the duodenum and the proximal sleeve margin in the stomach. In most cases, the initial intubation procedure took less than five minutes, and no extra steps were needed to facilitate transpyloric passage of the assembly. The depth of assembly insertion was $22-32 \mathrm{~cm}$ from the nares. If the assembly failed to pass into the duodenum on the first attempt, it was withdrawn by $5-8 \mathrm{~cm}$ and then advanced again after a period of no less than five minutes. Once the assembly was in position, an intragastric bolus feed containing $\left[{ }^{13} \mathrm{C}\right]$ octanoic acid was given (infusion channel) over a period of 5-15 minutes. Antropyloric motility was recorded from the start of feeding. Of the 43 studies attempted, recordings from 14 could not be analysed (PMA 30-33 weeks, $\mathrm{n}=1$; 34-36 weeks, $\mathrm{n}=10 ; 37-38$ weeks, $\mathrm{n}=3$ ). The main reasons for the failures were difficulties with initial transpyloric intubation, loss of an adequate TMPD signal, and displacement of the sleeve from the pyloric region such that antral pressures were recorded by an insufficient number of side holes. The failures occurred early in the study when we were developing expertise in the technique for positioning the manometric assembly.

\section{Analysis of manometric recordings}

All antropyloric pressure waves with peak amplitudes above a threshold of $5 \mathrm{~mm} \mathrm{Hg}$ were identified and analysed subject to confirmation of sleeve sensor position across the pylorus by TMPD. Antropyloric pressure waves were easily distinguishable from duodenal pressure waves by their longer duration (two to three seconds) and lower contraction frequency ( $1-5 /$ min). Abdominal straining was indicated by an instantaneous rise in pressure across all channels. IPPWs and PWSs were characterised according to criteria based on those previously used in adults, described below. ${ }^{8}$

\section{IPPWs}

These waves were defined as being isolated to the sleeve and/or one side hole occurring independently in time (10 seconds or more before to 10 seconds or more after) of any other pressure waves either antral or duodenal (fig 2A).

\section{Antropyloric PWSs}

These were defined as a series of two or more temporally related pressure waves with an onset either 0-10 seconds before or $0-10$ seconds after each other, but spatially separated-that is, recorded by different side holes. PWSs could include any combination of side holes that did not necessarily reach the most distal side hole or sleeve. Analysis of 
A

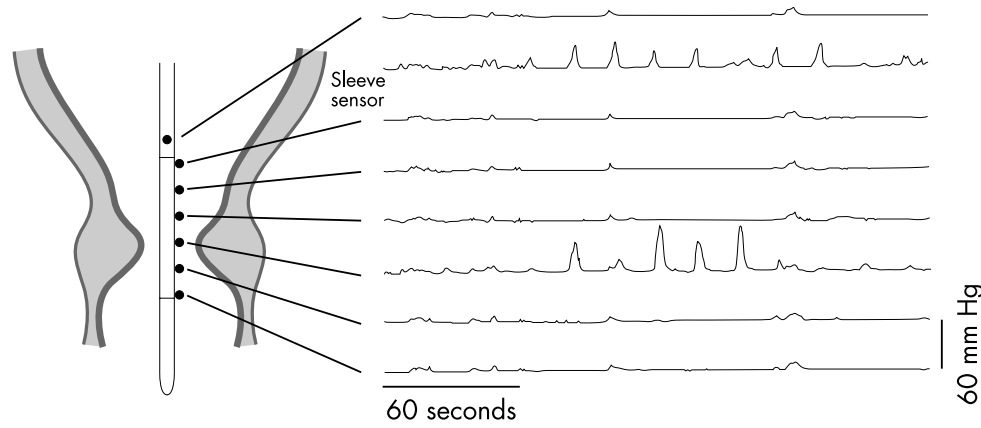

B

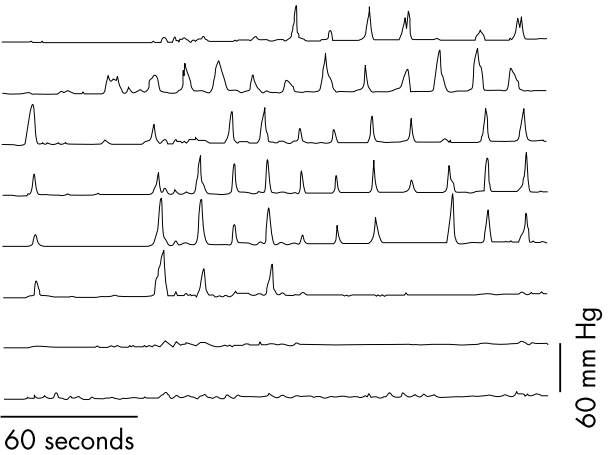

Figure 2 Example tracings showing $(A)$ isolated pyloric pressure waves and $(B)$ antegrade pressure wave sequences.

PWSs always began from the onset of the first pressure wave recorded regardless of its position within the recording area.

Four categories of PWSs were distinguished on the basis of spatial patterning and direction of the time of onset for individual pressure waves. These were: (a) synchronous PWSs where the time difference between the onset of two or more waves was less than one second; $(b)$ antegrade PWSs where the time difference between two or more pressure waves was 1-10 seconds and the direction of this difference aborad (shown in fig 2B); (c) retrograde PWSs, where the time difference between two or more pressure waves was $1-10$ seconds and the direction of this difference was orad; $(d)$ antegradesynchronous PWSs, where the sequence was made up of a combination of antegrade and synchronous. All motor patterns that did not meet the above criteria for IPPWs of PWSs were collectively grouped as other events.

\section{Statistical analysis}

Normally distributed data were expressed as mean (SEM), and skewed data were expressed as median (interquartile range). Relations between gastric emptying, feed volume, and pressure events were determined by simple regression statistics. Proportionate data were compared by the $\chi^{2}$ test. $\mathrm{p}<0.05$ was considered significant.

\section{RESULTS}

All infants showed IPPWs and all other types of motor patterns. A total of 7289 separate antropyloric motor patterns were analysed. IPPWs were the most common single motor pattern type observed (48\%), PWSs were predominantly antegrade $(18 \%)$, synchronous $(12 \%)$, or antegrade-synchronous $(13 \%)$, and retrograde PWSs $(2 \%)$ and other motor patterns (7\%) were least common.

The postprandial occurrence of IPPWs and PWSs differed considerably (fig 3 ). IPPWs were the single most common motor pattern recorded during the early postprandial period in all age groups. Feeding appeared to stimulate IPPWs and inhibit all other motor patterns (fig 3). In those infants $>33$ weeks who were studied for a long enough time, the occurrence of IPPWs peaked at 30-40 minutes after the start of feeding. In contrast with IPPWs, PWSs were more common during the second hour. The increase in the number of PWSs recorded in the second hour was due primarily to an increase in antegrade and antegrade-synchronous PWSs (8\% $v 18 \%$, $\mathrm{p}<0.05$ and $11 \% v 25 \%, \mathrm{p}<0.05$ respectively in the first $v$ second hour), whereas synchronous, retrograde, and other PWSs were not significantly changed ( $15 \% v 9 \%, 2 \% \vee 2 \%$, and $9 \% v 6 \%$ respectively in the first $v$ second hour).

The mean (SEM) half gastric emptying time was 42 (4) minutes, which coincided with the approximate time that IPPWs peaked (fig 3). The number of motor events recorded
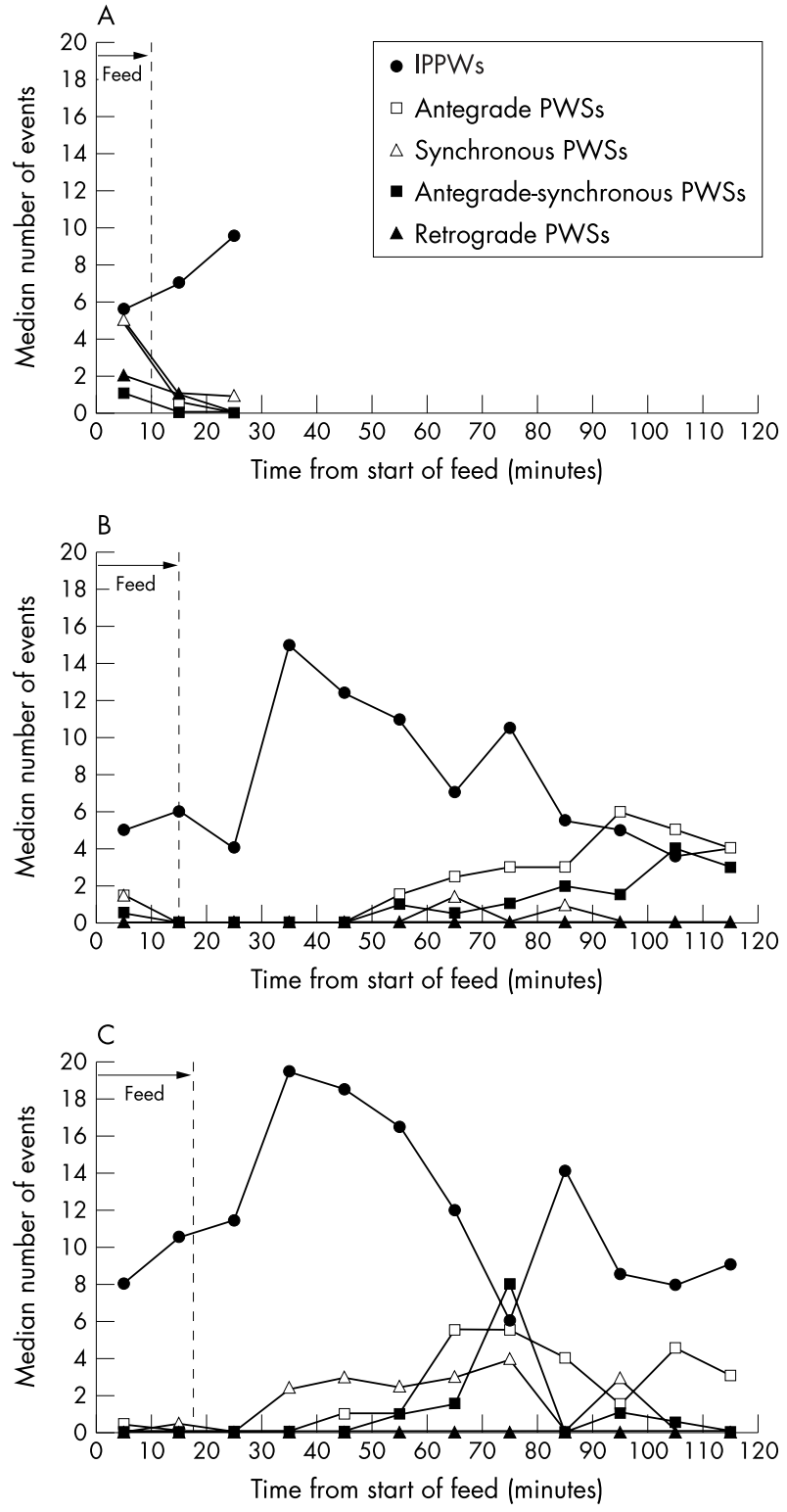

Figure 3 Occurrence of the antropyloric motor patterns over time in premature infants of ages 30-33 weeks (A), 34-36 weeks (B), and 37-38 weeks (C). The average time to complete a feed for each age group is indicated by the dotted line on each graph. Note that studies in the youngest infants were only performed for 30 minutes from the start of feeding. IPPW, isolated pyloric pressure wave; PWS, pressure wave sequence. 
$0-30,30-60,60-90$, and 90-120 minutes after the start of feeding were compared with half gastric emptying times and feed volumes ( $\mathrm{ml}$ and $\mathrm{ml} / \mathrm{kg}$ ). Significant correlations between these variables were only observed during the time interval 30-60 minutes, when faster gastric emptying correlated with greater numbers of antegrade PWSs $(r=0.421, \mathrm{p}<0.05)$, but not IPPWs or other PWSs, and larger feed volumes correlated with greater numbers of IPPWs $(r=0.36, \mathrm{p}=0.09 v \mathrm{ml}$ and $r=0.417, \mathrm{p}<0.05 v \mathrm{ml} / \mathrm{kg}$ ) and fewer antegrade PWSs $(r=0.670, \mathrm{p}<0.0005 v \mathrm{ml}$ and $r=0.568, \mathrm{p}<0.005 v \mathrm{ml} / \mathrm{kg})$, but not other types of PWS.

\section{DISCUSSION}

In this study, novel micromanometric techniques and breath test methods were used to characterise fed patterns of antropyloric motility during gastric emptying in healthy premature infants. The manometric data show a complex patterning of pressure events including IPPWs and antral PWSs which are very similar in appearance and occurrence to those described in normal healthy adult subjects. ${ }^{8}$

Earlier manometric studies have examined developmental aspects of gastrointestinal motility in premature infants, ${ }^{7-9}$ but these focused predominantly on the motility of the small intestine and were limited in their interpretation of antral and pyloric motor function because of the restricted number of recording side holes available at that time. Data from these early studies were reported as motility indices and, because of the small number of side holes available, it was not possible to evaluate pressure wave patterning in the same way as achieved in this study.

Using an array of seven closely spaced (3-5 mm) side holes straddling a total distance of $2 \mathrm{~cm}$, we were able to evaluate the spatial and temporal organisation of contractions in the pyloric region. We believe that these contractions are functionally important, working in conjunction with proximal gastric tone to regulate gastric emptying, producing either mixing or expulsion of stomach contents. ${ }^{16}$ Our measurements allowed us to describe four major subtypes of complex antral pressure wave patterning. These patterns were defined as "pressure wave sequences", a group of pressure waves which may contribute to a single contraction within the antropyloric region. ${ }^{8}$ This definition allowed us to assume that luminal flow was altered with each sequence recorded.

It has been well documented that the pylorus has an important role in the control of gastric emptying. ${ }^{16}{ }^{17}$ Our study is the first to record pressure waves isolated at the pylorus (IPPWs) in premature infants using a sleeve sensor, where accurate positioning of the sleeve was enabled by continuous monitoring of antroduodenal TMPD. In adults, IPPWs occur in response to a meal and/or duodenal nutrient infusion, indicating that IPPWs are stimulated by duodenal feedback mechanisms which concurrently inhibit antral and duodenal contraction in the region of the gastric outlet. ${ }^{17}$ Their predominance in the first postprandial hour, the time during which more than $50 \%$ of stomach contents was emptied, indicates that they are an important regulator of liquid emptying. With each IPPW, the gastric outlet is closed and transpyloric flow is obstructed for a period of two to three seconds. IPPWs are therefore essential for the regulated delivery of nutrients to the duodenum, and, without them, gastric emptying may proceed too quickly.

In this study, we were unable to show a clear relation between the occurrence of particular pressure events, such as IPPWs, and the rate of gastric emptying. This is perhaps not surprising given the complexity of the mechanisms involved and our still limited understanding of how gastric emptying is controlled by the subtle interplay between fundic tone and antropyloroduodenal motility. What was apparent, however, was that infants receiving proportionately greater feed volumes also had more IPPWs and fewer PWSs in the period 30-60 minutes after feeding, corresponding to the time point of half gastric emptying in most of the infants studied. These observations represent normal physiological responses to differences in meal size that would be predicted from previous studies in adults, ${ }^{18}$ larger liquid feeds in this case inhibiting PWSs and initiating more IPPWs to better control delivery of a greater volume of nutrient to the duodenum. These observations as a whole indicate that duodenal feedback mechanisms and antropyloric mechanisms regulating gastric emptying are functioning normally in premature infants.

Six infants 30-33 weeks PMA were only studied for 30 minutes and therefore it was only really possible to perform comparisons across all three age groups for data in the first 30 minutes. Our data nevertheless show that even the youngest premature infants studied exhibited what appeared to be a normal fed response of antral inhibition and stimulation of IPPWs.

In this study, we exclusively evaluated fed motor patterns because we felt that the literature was deficient in this area, namely in the characterisation of IPPWs and high resolution monitoring of antral PWSs in close proximity to the pyloric opening. Fasting motor patterns were not evaluated because all infants studied had already started regular bolus feeding. To obtain fasting motor events, these infants would need to be fasted for a period of eight or more hours to identify all three phases of the migrating motility complex. It was decided that such a protocol would be unnecessarily disruptive to the infants' feeding routine, far less acceptable to parents, and unlikely to provide any more information on the development of the migrating motor complex than already reported. ${ }^{19}$

In conclusion, our data clearly indicate that neuroregulatory mechanisms responsible for the coordination of antropyloric motility and gastric emptying are well developed in the human infant by 30 weeks PMA. Micromanometric techniques can now be used to investigate the gastric motor abnormalities likely to be associated with feed intolerance, reflux disease, intrauterine growth restriction, and severe prematurity.

\section{ACKNOWLEDGEMENTS}

We thank Mrs Ros Lontis and Mrs Louise Goodchild for their assistance with patient recruitment, providing routine bedside care, and for collecting the breath samples used for gastric emptying measurements, and Professor Charles Malbert for the data acquisition and analysis program used in this study.

\section{Authors' affiliations}

B B Hassan, R Butler, G P Davidson, T I Omari, Centre for Paediatric and Adolescent Gastroenterology, Women's and Children's Hospital, North Adelaide, Australia

R Haslam, C Barnett, Department of Neonatal Medicine, Women's and Children's Hospital, North Adelaide

M Benninga, Department of Pediatric Gastroenterology and Nutrition, Academic Medical Centre, Amsterdam, The Netherlands

J Dent, Department of Gastrointestinal Medicine, Royal Adelaide Hospital, Adelaide, Australia

G P Davidson, T I Omari, Department of Paediatrics, University of Adelaide, Adelaide, Australia

\section{REFERENCES}

1 Dumont RC, Rudolph CD. Development of gastrointestinal motility in the infant and child. Gastroenterol Clin North Am 1994;23:655-71.

2 Cavill B. Gastric emptying in preterm infants. Acta Paediatr Scand 1979;68:725-30.

3 Newell SJ, Chapman S, Booth IW. Ultrasonic assessment of gastric emptying in the preterm infant. Arch Dis Child 1993;69:32-6.

4 Cucchiara S, Salvia G, Scarcella A, et al. Gestational maturation of electrical activity of the stomach. Dig Dis Sci 1999;44:2008-13.

5 Berseth CL. Neonatal small intestinal motility: motor responses to feeding in term and preterm infants. J Pediatr 1990;117:777-82. 
6 Berseth CL, Ittmann PI. Antroduodenal motor responses to duodenal feeding in preterm and term infants. J Pediatr Gastroenterol Nutr 1992; 14:182-6.

7 Berseth CL. The effect of early feeding on maturation of preterm infant's small intestine. J Pediatr 1992;120:947-53.

8 Sun WM, Hebbard GS, Malbert $\mathrm{CH}$, et al. Spatial patterns of fasting and fed antro-pyloric pressure waves in humans. J Physiol 1997:503:455-62.

9 Omari, Tl, Miki K, Davidson GP, et al. Characterisation of relaxation of the lower oesophageal sphincter in healthy premature infants. Gut 1997;40:370-5

10 Omari TI, Barnett C, Snel A, et al. Mechanisms of gastro-oesophageal reflux in healthy premature infants. J Pediatr 1998;133:650-4.

11 Heddle R, Fone D, Dent J, et al. Stimulation of pyloric motility by intraduodenal dextrose in normal subjects. Gut 1988;29:1349-57.

12 Barnett C, Snel A, Omari T, et al. Reproducibility of the ${ }^{13} \mathrm{C}$-octanoic acid breath test for assessment of gastric emptying in healthy preterm infants. J Pediatr Gastroenterol Nutr 1999;29:26-30.
13 Veereman-Wauters G, Ghoos Y, Van der Schoor S, et al. The ${ }^{13} \mathrm{C}$-octanoic acid breath test: a non-invasive technique to assess gastric emptying in preterm infants. J Pediatr Gastroenterol Nutr 1996;23:111-17

14 Van Den Driessche M, Peeters K, Marien P, et al. Gastric emptying in formula-fed and breast-fed infants measured with the ${ }^{13} \mathrm{C}$-octanoic acid breath test. J Pediatr Gastroenterol Nutr 1999:29:46-51.

15 Ghoos YF, Maes BD, Geypeyns BJ, et al. Measurement of gastric emptying rate of solids by means of a carbon-labelled octanoic acid breath test. Gastroenterology 1993;104:1640-7.

16 Horowitz M, Dent J. The study of gastric mechanics and flow: a Mad Hatter's tea party starting to make sense? Gastroenterology 1994; 107:302-6.

17 Raiser F, Hinder R. The importance of the pylorus in gastric emptying. J Gastroenterol Hepatol 1995;10:666-7.

18 Stanghellini V, Borovicka J, Read N. Feedback regulation and sensation. Dig Dis Sci 1994;39:124-7S.

19 Ittmann PI, Amarnath R, Berseth CL. Maturation of antroduodenal motor activity in preterm and term infants. Dig Dis Sci 1992;37:14-19.

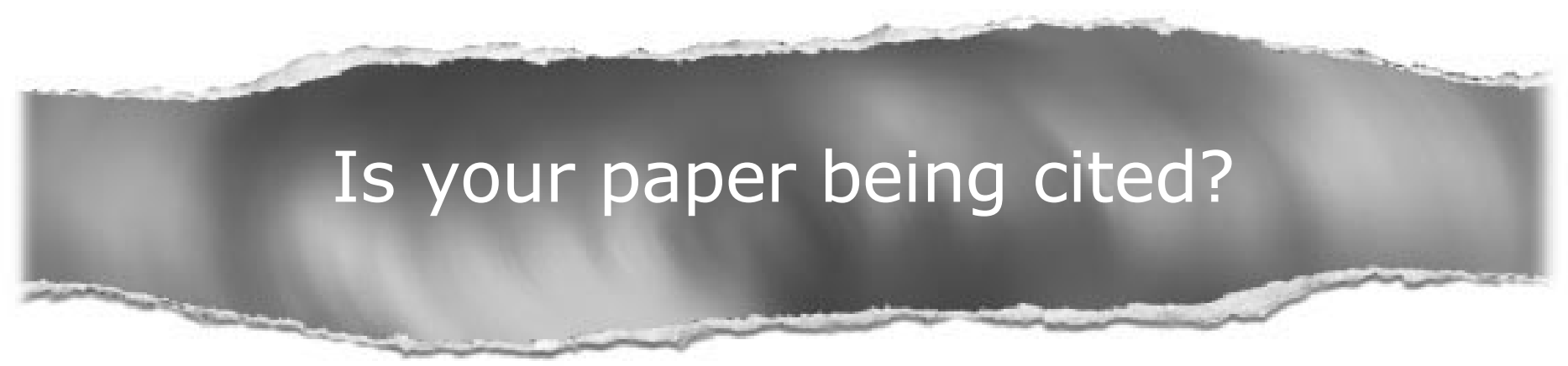

\section{CiteTrack service}

CiteTrack will alert you by email whenever new content in Archives of Disease in Childhood or a participating journal is published that matches criteria you want to track

Topics: Tell CiteTrack which words or subjects to watch for in new content Authors: Be alerted whenever key authors you are following publish a new paper Articles: Know whenever a paper of interest to you is referenced by another paper

\section{www.archdischild.com}

